\title{
Fluid-fluid level on magnetic resonance images may predict the occurrence of pituitary adenomas in cystic sellar-suprasellar masses
}

\author{
DEYONG XIAO $^{1}$, SHOUSEN WANG $^{1}$, LIN ZHAO $^{1}$, QUN ZHONG $^{2}$, YINXING HUANG $^{1}$ and CHENYU DING $^{1}$ \\ Departments of ${ }^{1}$ Neurosurgery and ${ }^{2}$ Radiology, Fuzong Clinical College, \\ Fujian Medical University, Fuzhou, Fujian 350025, P.R. China
}

Received November 17, 2015; Accepted December 23, 2016

DOI: $10.3892 /$ etm.2017.4299

\begin{abstract}
The aim of the present study was to evaluate the value of identifying fluid-fluid level via preoperative magnetic resonance (MR) images in differentiating pituitary adenomas from craniopharyngioma, Rathke's cleft cyst (RCC), and other cystic sellar-suprasellar lesions. The data of 293 consecutive patients who underwent surgery for sellar-suprasellar lesions between July 2010 and December 2012 was retrospectively reviewed, and a total of 133 cystic cases were included in the present study. MR images and pathological features of all subjects were examined. Among the 133 cystic sellar-suprasellar masses, there were 78 cases of pituitary adenomas, 31 cases of craniopharyngioma, 21 cases of RCC, one case of epidermoid cyst and two cases of abscess. Fluid-fluid levels were identified n 43 cases $(55.13 \%)$ of cystic pituitary adenomas, with a single fluid level in 23 cases, two fluid levels in 8 cases, and three levels or more in 12 cases. Two cases $(6.45 \%)$ of craniopharyngioma, and one case $(4.76 \%)$ of RCC presented single fluid level. No instances of fluid-fluid levels were observed in epidermoid cyst or abscess. Fluid-fluid levels were typically exhibited on axial T2-weighted images. The identification of fluid-fluid level in cysts provides useful diagnostic value in distinguishing pituitary adenoma from other sellar-suprasellar lesions. The findings of the present study suggest that a sellar-suprasellar mass with a fluid-fluid level inside the tumor is most likely a pituitary adenoma, particularly if multiple fluid levels are observed in the same tumor. The fluid-fluid level is typically clearly depicted on axial or sagittal MR images, suggesting subacute or chronic hemorrhage in pituitary adenomas.
\end{abstract}

Correspondence to: Dr Shousen Wang, Department of Neurosurgery, Fuzong Clinical College, Fujian Medical University, 156 Xihuanbei Road, Fuzhou, Fujian 350025, P.R. China

E-mail: xiaody76@163.com

Key words: pituitary neoplasms, magnetic resonance imaging, differential, fluid-fluid level

\section{Introduction}

The sellar and suprasellar regions are anatomically complex areas where a number of neoplastic, congenital, inflammatory and infectious lesions may occur (1). Pituitary adenomas are the predominant cause of these lesions (2). Other causes include Rathke's cleft cyst (RCC), craniopharyngioma, meningiomas, germ cell tumors, gliomas, dermoid and epidermoid tumors and pituitary abscess (2). As surgical planning or treatment is different for each of these diseases, it is important for neurosurgeons to make accurate diagnosis prior to surgery. Although many sellar and suprasellar lesions maybe distinguished by their relevant magnetic resonance (MR) imaging (MRI) and computed tomography (CT) characteristics, it is not always easy to make a differential diagnosis when a lesion occurs in both the intrasellar and suprasellar regions, particularly when the lesion has solid and cystic, or cystic characteristics (3).

Fluid-fluid levels have been observed in various benign or malignant tumors, such as bone and soft tissue tumors, schwannomas, and sarcomas $(4,5)$. Nevertheless, few articles have reported fluid-fluid levels in sellar pathologic masses, and the value of fluid-fluid levels in the differential diagnosis of sellar lesions is rarely reported. Catalano et al (6) have previously stated that fluid-fluid levels are a result of the separation of unclotted blood as opposed to serous fluid, and that MRI is more sensitive than CT to detect fluid-fluid levels due to its better contrast resolution and sensitivity for subacute and chronic hemorrhage. In the present study, the sectional imaging characterization of fluid-fluid levels on MRI and its value for preoperative differential diagnosis in cystic sellar-suprasellar lesions were evaluated.

\section{Materials and methods}

Patients. The clinical data of 293 consecutive patients who received initial surgery for sellar-suparsellar lesions in the Department of Neurosurgery at Fuzhou General Hospital (Fuzhou, China) between July 2010 and December 2012 were retrospectively reviewed. Among the 293 patients, 133 cases were included in the present study, including 78 cases of cystic pituitary adenoma, 31 cases of craniopharyngioma, 21 cases of RCC, two cases of pituitary abscess and one case of epidermoid 
cyst. The inclusion criteria were as follows: i) Tumors in the sellar and suparsellar regions; ii) tumors identified as having solid and cystic, or cystic characteristics via preoperative MRI; iii) cyst or cystic fluid is confirmed during operation; and iv) diagnosis was confirmed via surgery and pathology. All subjects were confirmed to have sellar-suparsellar masses via surgery and pathology. The exclusion criteria were as follows: i) Substantive pituitary adenomas $(n=106)$; ii) meningiomas $(n=26)$ and germ cell tumors $(n=5)$; iii) tumors limited to sellar region ( $n=2)$; iv) MRI was not performed or MR images were not available $(n=3)$; and v) surgery was performed prior to MRI ( $n=18)$. All the procedures were approved by the Ethics Committee of Fujian Medical University. Written informed consent was obtained from all patients or their families.

MRI. In 19 patients, MR images were obtained with a $1.5-\mathrm{T}$ MRI scanner (SIGNA; GE Healthcare, Chalfont, UK), and the other 114 patients were examined using a3.0-T MRI machine (Siemens Healthcare, Erlangen, Germany). Imaging sequences included at least axial T2-weighted, sagittal and coronal T1-weighted, and contrast-enhanced T1-weighted sagittal and coronal sequences, which were obtained at 30-60 sec following an intravenous bolus injection of $0.1 \mathrm{mmol} / \mathrm{kg}$ gadopentetatedime glumine (Magnevist; Bayer AG, Leverkusen, Germany). Section thickness was $5 \mathrm{~mm}$ in axial and $2.5-3 \mathrm{~mm}$ in sagittal or coronal sequences, with a 6-7 $\mathrm{mm}$ intersection gap in axial and $2.75-3 \mathrm{~mm}$ intersection gap in sagittal or coronal sequences. Pre- and post-contrast T1-weighted images were obtained at 400-600/8-24 msec (repetition time/echo time) with two or three signals being acquired. T2-weighted fast spin-echo images were obtained at 3,000-4,000/80-150 msec with 2-3 signals being acquired. Preoperational MR images were evaluated together by two neurosurgery operators and one radiological associate chief physician, to conduct a detailed analysis and provide consistent opinions about tumor cystic degeneration and the imaging features of fluid-fluid levels in cystic masses. The MR signal of super-/sub-component of fluid-fluid levels was compared with cerebral gray matter and described as: Hyper-/iso-/hypo-intense.

Pathology and immunohistochemistry. Hematoxylin and eosin staining and immunohistochemical examination were carried out for etiological diagnosis in the Department of Pathology at Fuzhou General Hospital (Fuzhou, China). The specific steps of immunohistochemistry were as follows. Surgical resection specimens were fixed and embedded in paraffin. Paraffin-embedded tissue was sliced into $4-\mu \mathrm{m}$ sections, deparaffinized using xylene and rehydrated in graded ethanol (100, 85 and $75 \%$, each for $2 \mathrm{~min})$. Antigen retrieval was performed by pressure cooking in a $\mathrm{pH} 6$ citrate buffer with Citrate Tissue Antigen Buffer (Fuzhou MaixinBiological Technology Co., Ltd., Fuzhou, China) and endogenous peroxidase activity was blocked with hydrogen peroxide. Non specific binding was blocked by incubating slides with goat serum. Primary antibody (Rabbit anti-Prolactin (RAB-0109), Rabbit anti-Growth Hormone (RAB-0084), Mouse anti-Luteinizing Hormone (MAB-0114), Mouse anti-FSH (MAB-0204), Mouse anti-TSH (MAB-0162); dilution 1:200; Fuzhou MaixinBiological Technology Co., Ltd., Fuzhou, China) was added and incubated with tissue for $2 \mathrm{~h}$ at room temperature.
The sections were incubated with rapid enzyme-labeled goat anti-mouse/rabbit IgG polymers (catalogue no. KIT-5020; Fuzhou MaixinBiological Technology Co., Ltd., Fuzhou, China) for $30 \mathrm{~min}$ at $37^{\circ} \mathrm{C}$. Immunoreactivity was visualized using 3,3'diamino-benzidine. Examination was performed under a light microscope using 100-400x magnification. In the negative control, PBS was used instead of the primary antibody.

The immunohistochemical subtype of pituitary adenoma was determined according to the 2004 World Health Organization immunohistochemical classification standard (7) with regard to the following: Prolactin (PRL), growth hormone (GH), adrenocorticotropic hormone (ACTH), thyroid-stimulating hormone (TSH), follicle-stimulating hormone (FSH) and luteinizing hormone (LH).

Statistical analysis. Statistical analyses were performed using SPSS 13.0 software (SPSS, Inc., Chicago, IL, USA). Chi-square tests were performed to compare the incidence rate of fluid-fluid levels. $\mathrm{P}<0.05$ was considered to indicate a statistically significant difference.

\section{Results}

Fluid-fluid levels are common in cystic pituitary adenomas. To investigate the association between fluid-fluid levels and pituitary adenomas, preoperational MRIs and surgical pathology results were analyzed. The data showed that 78 cases $(42.39 \%)$ of pituitary adenomas diagnosed within this period exhibited evidence of cystic degeneration within the tumor. A total of 43 cases presented fluid-fluid level formation, including 23 cases of single level, 8 cases of two levels, and 12 cases of three levels or higher (Table I). Fluid levels were observed in $55.13 \%$ of patients with cystic pituitary tumors via MRI, and tend to be non-functional, FSH/LH, PRL, and multi-functional pituitary adenomas (Tables I and II). In T2-weighted images, the super-/sub-component of fluid-fluid levels in 43 cases of cystic pituitary adenomas included 32 hyper-/hypo-intense cases, 10 hyper-/iso-intense cases, and 1hypo-/iso-intense case. However, these levels were only observed in the T1-weighted images of 12 cases, including eight hyper-/iso-intense cases, 2 hyper-/hypo-intense cases, 1 slightly hyper-/hyper-intense case, and 1 slightly hypo-/iso-intense case (Table II). In the same cohort of cystic pituitary adenomas, various levels exhibited similar signal characteristics on MRI. Cystic areas without fluid-fluid level formation produced low or slightly low signals in T1-weighted images and high signals in T2-weighted images (Figs. 1 and 2). All pituitary adenomas were resected via trans sphenoidal surgical route under a microscope. During surgery, the cyst fluid was thick and dark red in the pituitary masses and exhibited fluid-fluid levels on MRI, whereas thin xanthochromic or clear fluid was observed in those which did not exhibit fluid-fluid levels on MRI. These results suggest that the signs of fluid-fluid level are more common in pituitary adenomas, suggesting subacute or chronic hemorrhage in pituitary adenomas.

Fluid-fluid levels are rare in non-pituitary adenoma lesions of the sellar-suprasellar region. To investigate the association between fluid-fluid levels and non-pituitary adenoma causes 
Table I. Immunohistochemical indicators and fluid-fluid levels in 78 cases of cystic pituitary adenomas.

\begin{tabular}{|c|c|c|c|c|c|}
\hline \multirow[b]{2}{*}{ Immunohistochemistry } & \multirow[b]{2}{*}{ Cases (n) } & \multicolumn{4}{|c|}{ Fluid-fluid levels (n) } \\
\hline & & None & 1 & 2 & $\geq 3$ \\
\hline PRL & 18 & 9 & 6 & 2 & 1 \\
\hline FSH/LH & 25 & 13 & 7 & 2 & 3 \\
\hline АCTH & 3 & 2 & 0 & 0 & 1 \\
\hline GH & 1 & 1 & 0 & 0 & 0 \\
\hline TSH & 0 & 0 & 0 & 0 & 0 \\
\hline Non-functional & 24 & 6 & 8 & 4 & 6 \\
\hline Multi-functional & 7 & 4 & 2 & 0 & 1 \\
\hline Total & 78 & 35 & 23 & 8 & 12 \\
\hline
\end{tabular}

PRL, prolactin; FSH, follicle-stimulating hormone; LH, luteinizing hormone; ACTH, adrenocorticotropic hormone; GH, growth hormone; TSH, thyroid-stimulating hormone.

Table II. Fluid-fluid levels in cystic pituitary adenomas, craniopharyngioma, and RCC that affect sellar and suprasellar regions.

\begin{tabular}{|c|c|c|c|c|c|}
\hline Fluid-fluid levels & Pituitary adenoma $(\mathrm{n}=78)$ & Craniopharyngioma $(\mathrm{n}=31)$ & $\operatorname{RCC}(n=21)$ & $\chi^{2}$ & P-value \\
\hline \multicolumn{6}{|l|}{ Level No. } \\
\hline $1, \mathrm{n}(\%$ of total $)$ & $23(29.5 \%)$ & $2(6.45 \%)$ & $1(4.76 \%)$ & & \\
\hline $2, \mathrm{n}(\%$ of total $)$ & $8(10.3 \%)$ & & & & \\
\hline$\geq 3, \mathrm{n}(\%$ of total $)$ & $12(15.4 \%)$ & & & & \\
\hline Total, n (\% of total) & $43(55.13 \%)$ & & & 33.261 & $<0.001$ \\
\hline \multicolumn{6}{|l|}{ Super-/sub-signals on MRI } \\
\hline \multicolumn{6}{|l|}{$\mathrm{T} 2 \mathrm{WI}$} \\
\hline Hyper-/hypo- & 32 & & & & \\
\hline Hyper-/iso- & 10 & & & & \\
\hline Hypo-/iso- & 1 & & & & \\
\hline \multicolumn{6}{|l|}{ T1WI } \\
\hline Hyper-/iso- & 8 & & & & \\
\hline Hyper-/hypo- & 2 & & & & \\
\hline Slightly hyper-/hyper- & 1 & & & & \\
\hline Slightly hypo-/iso- & 1 & & & & \\
\hline
\end{tabular}

RCC, Rathke's cleft cyst; WI, weighted image.

of lesions in sellar and suprasellar regions, patients with craniopharyngioma, RCC, epidermoid tumors, pituitary abscess, germ cell tumors and meningiomas were studied using MRI. The results showed that 31 cases of craniopharyngioma were mixed solid-cystic tumors. On T1-weighted and T2-weighted images, the cystic tumor parts were predominantly hyperintense or rarely hypointense (data not shown). Notably, the cystic portions of tumors in three patients were hypo-intense on T1-weighted images, and either hyper-intense or mixed (hyper- and hypo-intense) on T2-weighted images. A liquid-filled space with a fluid-fluid level inside the lesions was observed in two cases of craniopharyngioma, which exhibited hyperintense supernatants on T1- and T2-weighted images, and iso- and hypo-intense subnatants on T1- and T2-weighted images, respectively (Fig. 3). For these patients, dark red cyst liquids were expunged during surgery. There were 21 cases of RCC, and a single fluid-fluid level was found in one case via MRI, which was hyper-/hypo-intense on T2-weighted images and hyper-/iso-intense on T1-weighted images. In addition, evident intracystic nodules were observed in four patients (Fig. 4), which had homogenous intensity without enhancement. However, no fluid-fluid levels were identified on these MR images. In the present study, fluid-fluid levels were not identified in epidermoid cyst, pituitary abscess, germ cell tumors ormeningiomas. Two pituitary abscesses exhibited slightly increased T2-weighted signals and reduced T1-weighted signals on their MR images, with a thin rim of enhancement; however, no fluid-fluid level was observed in the cyst. The incidence of fluid-fluid level was $55.13 \%$ in cystic pituitary adenomas, which was significantly higher than in 


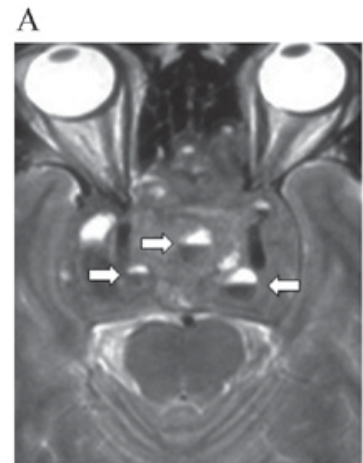

B
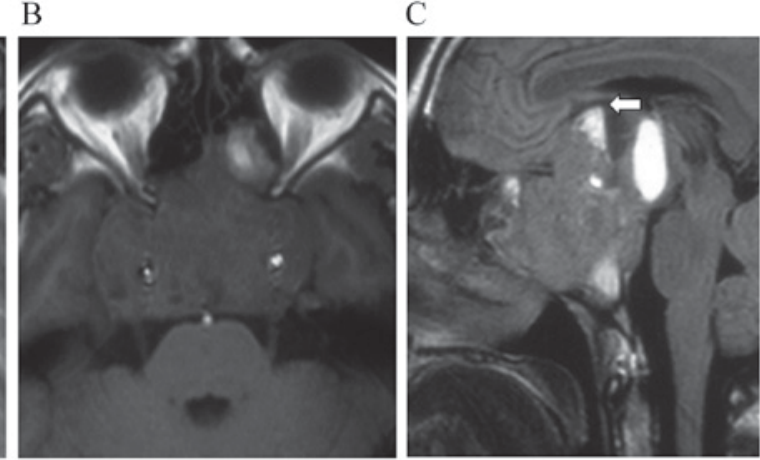

D

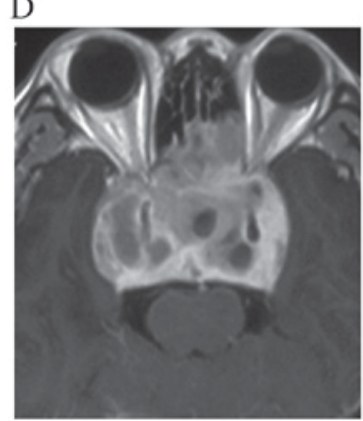

E

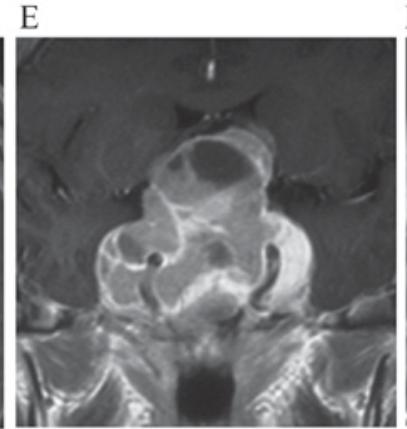

$\mathrm{F}$

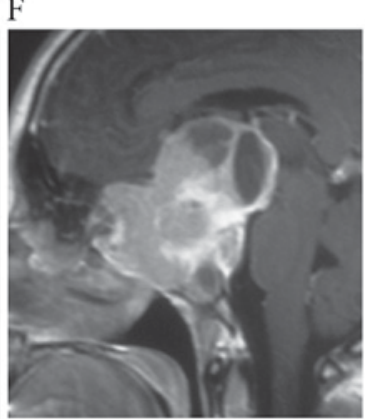

Figure 1. Magnetic resonance images of cystic prolactin pituitary adenoma with multiple fluid-fluid levels in a 38-year-old female. Arrows indicate fluid-fluid level. (A) T2-weighted image (axial). (B) T1-weighted image (axial). (C) T2 flair (sagittal). (D) Contrast-enhanced T1-weighted image (axial). (E) Contrast-enhanced T1-weighted image (coronal). (F) Contrast-enhanced T1-weighted image (sagittal).

A

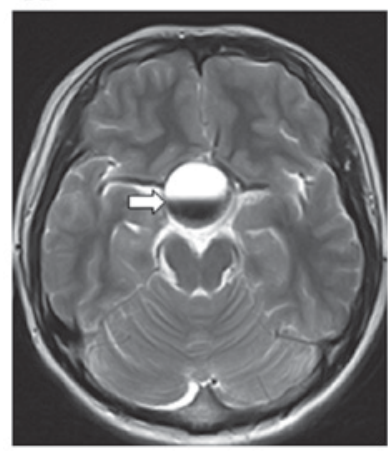

B

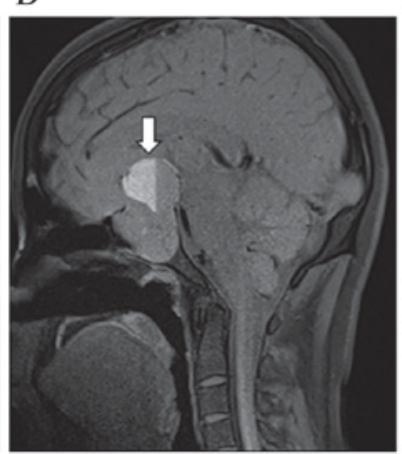

C

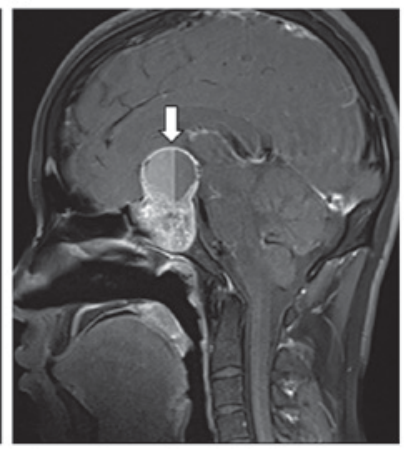

Figure 2. Magnetic resonance images of invasive pituitary adenoma with a fluid-fluid level in the supersellar cyst. (A) Axial T2-weighted image showing the super-/sub-component of fluid-fluid level as hyper-/hypo-intense. (B) Sagittal T1-weighted image showing the fluid-fluid level as hyper-/iso-intense. (C) Sagittal contrast-enhanced T1-weighted image showing snowman shape, and homogeneous enhancement of the solid portion. Fluid-fluid levels are indicated by arrows.

craniopharyngiomas $(6.45 \% ; 2 / 31)$ and RCCs $(4.76 \% ; 1 / 21$; $\mathrm{P}<0.01)$. These results suggest that sellar-suprasellar masses, which exhibit fluid-fluid levels inside the tumor, are most likely pituitary adenoma, particularly when multiple fluid levels are present in the same tumor.

\section{Discussion}

Sellar and suprasellar masses are varied in etiology and may cause diagnostic and management dilemmas for neurosurgeons. For example, distinguishing pituitary adenoma from craniopharyngioma, RCC, and other cystic sellar-supersellar masses may be challenging, as intra tumoral hemorrhage and cystic change are frequently observed in macro adenomas $(3,8,9)$. In the present study, fluid-fluid levels were detected in $55.13 \%$ of cystic pituitary adenoma cases; however, they were rarely observed in RCC, craniopharyngioma and pituitary abscess. Furthermore, multi-fluid level phenomenon was only observed in pituitary adenomas. In addition, as meningiomas and germ cell tumors are almost substantive, fluid-fluid levels should not be detected in these cases (2). Therefore, the identification of fluid-fluid levels may be beneficial in diagnosing pituitary adenomas and distinguishing them from other sellar-suprasellar lesions. The results of the present study suggest that a sellar-suprasellar mass with an intratumor fluid-fluid level is most likely a pituitary adenoma, particularly when multiple fluid levels are present in the same tumor.

Pituitary adenomas are the most prevalent lesions in the sellar-supersellar region. Cystic degenerations in pituitary adenomas are believed to be the result of hemorrhage or 

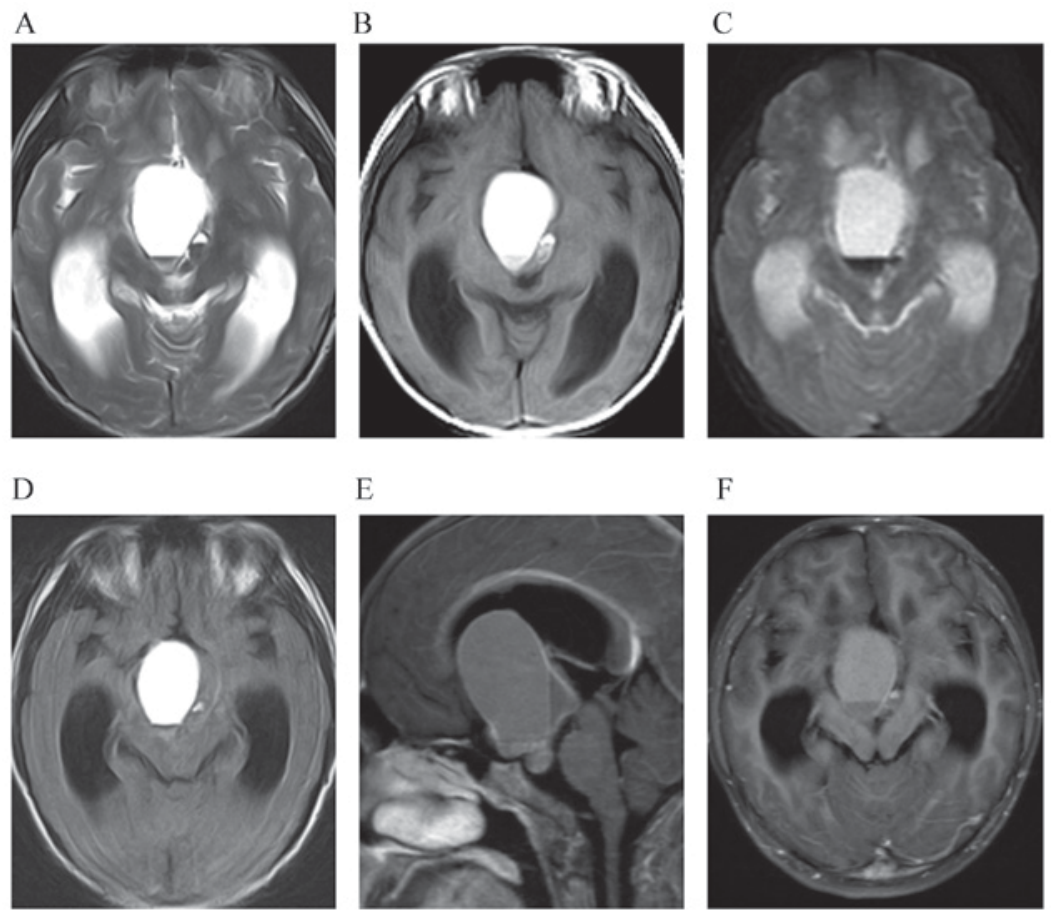

E

$\mathrm{F}$
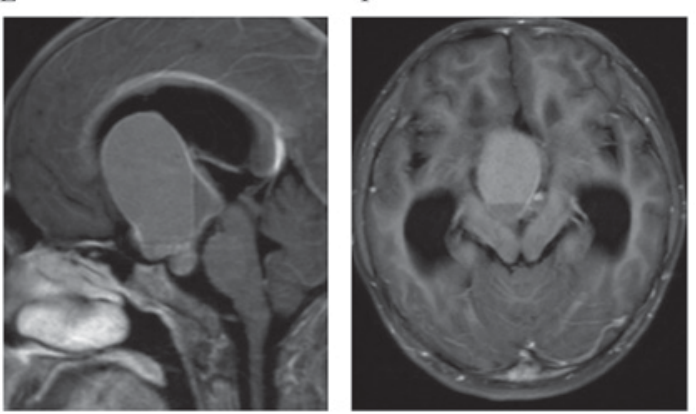

Figure 3. Magnetic resonance images of craniopharyngioma with a fluid-fluid level and third ventricle compression by superior tumor extension. (A) T2-weighted image (axial). (B) T1-weighted image (axial). (C) Diffusion-weighted image (axial). (D) T2 flair (axial). (E) Contrast-enhanced T1-weighted image (sagittal). (F) Contrast-enhanced T1-weighted image (axial). Dark red cyst liquids were expunged during surgery.

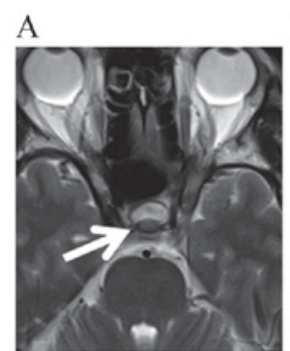

$\mathrm{E}$

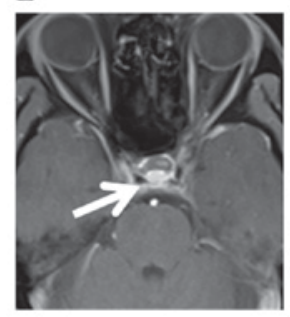

B

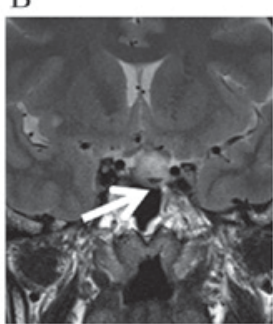

$\mathrm{F}$

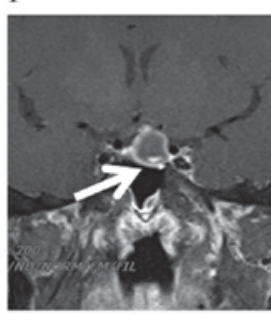

$\mathrm{C}$

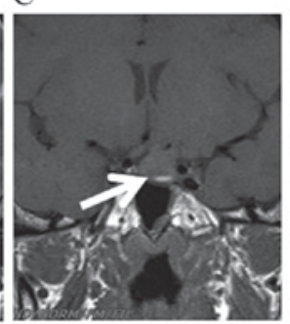

G

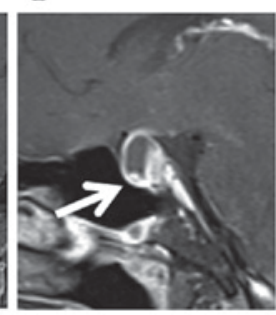

$\mathrm{D}$

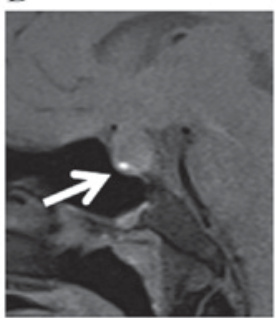

Figure 4. Magnetic resonance images of Rathke's cleft cyst with intracystic nodule in a 47-year-old male who suffered from headache for 3 weeks. (A) Axial and (B) coronal images exhibiting a low-signal intracystic nodule (arrow) on T2-weighted image with no fluid-fluid level. (C) Coronal and (D) sagittal images showing the nodule with high signal on T1-weighted image due to high protein. (E) Axial, (F) coronal and (G) sagittal contrast-enhanced T1-weighted images showing a well-demarcated sell-suprasellar cystic mess with thin cyst wall enhancement and intracystic nodule.

necrosis $(10,11)$. Macro adenomas typically extend into the suprasellar cistern and compress the optic chiasm; therefore, blood vessels maybe oppressed near the diaphragm sellae, leading to ischemic necrosis of the adenomas. Bleeding also typically occurs in pituitary adenomas. Repeated bleeding may occur on the basis of ischemic necrosis or hemorrhagic necrosis (11-13). Various previous studies have suggested that fluid-fluid level within a fluid cavity is induced by the sedimentation of blood products at the subacute or chronic stage, as well as the separation of unclotted blood as opposed to serous (interstitial) fluid $(6,10-12,14,15)$. These studies indicate that fluid-fluid levels are typically presented as high/low signal patterns on axial (or sagittal) T2-weighted images, or partially on T1-weighted images as high/slightly high (or isointense) features. Furthermore, the supernatant high-intense areas demonstrated xantho chromic fluid, and the subnatant low-intense areas demonstrated dark-red liquefied hematoma (10). In the present study, fluid-fluid levels within pituitary adenomas were well defined on T2-weighted images, and their MRI features were consistent with a 
hematoma-metabolic period (subacute or chronic stage), dark red cyst fluid was expunged during operation.

The incidence of fluid-fluid level in cystic pituitary adenomas was $55.13 \%$ in the present study. Non-functional FSH/LHPRL and multi-functional pituitary adenomas were typically shown to present fluid-fluid levels or cavities. These results are in accordance with the previous observation that the occurrence of fluid-fluid levels within hemorrhagic pituitary tumors ranges from 48.1-55.8\% $(10,14)$. Additionally, hemorrhage within pituitary adenomas is common in patients with non-functioning adenomas and prolactinomas $(9,16-21)$; although an earlier study suggests that GH-and ACTH-secreting pituitary adenomas are more likely to present apoplexy than others (22). This immunological variation may be due to the lack of specific clinical markers for non-functioning and FSH/LH tumors, which may remain undetected for many years until they become large enough to induce tumor ischemia $(13,21)$. In PRL and multi-functional pituitary adenomas, hemorrhage and cystic degeneration may occur due to high metabolism and relative ischemia (19). In addition, it is notable that large or rapidly growing pituitary adenomas maybe prone to hemorrhage and cyst formation (17).

RCC and craniopharyngioma are also common lesions in the sellar and suprasellar regions. On MRI, RCC is typically exhibited as an intrasellar cystic lesion with suprasellar extension that is ovoid or kidney shaped and well demarcated, and exhibits no calcification, a lack of or a thin cyst wall enhancement, orintracystic nodules (2). Craniopharyngiomas are typically presented as superiorly lobulated shaped sellar-suprasellar heterogeneous lesions, with mixed solid and cystic characteristics, calcification, reticular enhancement of the solid portion, and third ventricle compression by superior tumor extension $(8,15,23)$. In various previous studies, intracystic nodules have been regarded to have diagnostic value for RCC, which typically displays distinctive characteristics, including T1-weighted hyperintensity, T2-weighted hypointensity, and no gadolinium enhancement (24-26). However, it may be difficult to distinguish the intracystic nodule of RCC from acute hemorrhage observed in pituitary apoplexy. The nodules correspond to protein concretions and appear inside the cyst. However, the fluid-fluid level is due to the sedimentation of blood products and often appears in the suprasellar portion of the tumor (26). The present study suggests that the presence of a hemorrhagic fluid-fluid level in sellar-suprasellar lesions on MRI maybe used to differentiate pituitary adenomas from craniopharyngiomas and $\mathrm{RCC}$, as bleeding is rarely observed in craniopharyngiomas and RCC. Furthermore, axial or sagittal images are necessary to exclude the identification of a fluid-fluid level. Bonneville et al (15) have previously suggested that a fluid-fluid level is beneficial in differentiating adenoma from RCCs and craniopharyngiomas. To the best of our knowledge, only a few cases of bleeding in RCCs and craniopharyngiomas have been described, and it has been suggested that bleeding is a result of compressed portal veins or newly organized thin-wall vessels within the granulation tissue of the cyst wall, particularly in large cysts $(15,27)$. A pseudo-fluid-fluid level may be observed in craniopharyngiomas, corresponding to tenacious secretions or the position of the interface between the fluid and solid components of the tumor (26). Furthermore, diffusion-weighted MRI and relative apparent diffusion coefficient, as well as clinical presentation and endocrine appearance, have been suggested to be beneficial in differentiating among pituitary adenomas, RCCs, craniopharyngiomas and other cystic sellar lesions (28).

Pituitary abscess is very rare (2). It typically appears as round sellar masses with characteristic rim enhancement and associated adjacent inflammatory reaction. Hemorrhagic fluid-fluid level has been rarely described in pituitary abscess. Furthermore, meningeal enhancement induced by concurrent meningitis may help distinguish pituitary abscess from pituitary adenoma (2). However, the present study has several limitations. Firstly, other infrequent sellar-suprasellar lesions, such as arachnoid cysts, dermoid cysts and epidermoid cysts were not included in the present study. Second, the sample size of non-adenomatous lesions is relatively small.

In conclusion, fluid-fluid level formation is a nonspecific imaging finding that may be encountered in non-adenomatous sellar-suprasellar lesions that highly suggest hemorrhagic pituitary adenoma. These findings suggest that in clinical practice, pituitary adenoma should be considered initially following the identification of marked cystic formation with fluid-fluid levels in the sellar-suprasellar mass via MRI. Furthermore, hemorrhagic pituitary adenoma should be considered if the hematomais at the subacute or chronic stage, and axial T2-weighted images are beneficial in identifying fluid-fluid levels.

\section{Acknowledgements}

The authors of the present study are grateful to the Department of Pathology at Fuzhou General Hospital (Fuzhou, China) for their pathological examination and assessment. The present study was supported by the Medical Science Innovation Research Fund of Nanjing Military Region, China (grant no. 11Z034).

\section{References}

1. Rennert $\mathbf{J}$ and Doerfler A: Imaging of sellar and parasellar lesions. Clin Neurol Neurosurg 109: 111-124, 2007.

2. Connor SE and Penney CC: MRI in the differential diagnosis of a sellar mass. Clin Radiol 58: 20-31, 2003.

3. Kitajima M, Hirai T, Katsuragawa S, Okuda T, Fukuoka H, Sasao A, Akter M, Awai K, Nakayama Y, Ikeda R, et al: Differentiation of common large sellar-suprasellar masses effect of artificial neural network on radiologists' diagnosis performance. Acad Radiol 16: 313-320, 2009.

4. Kato H, Kanematsu M, Mizuta K, Aoki M, Yamada K, Yamakawa H, Iwama T and Hirose Y: Fluid-fluid level formation: A rare finding of extracranial head and neck schwannomas. AJNR Am J Neuroradiol 30: 1451-1453, 2009.

5. Van Dyck P, Vanhoenacker FM, Vogel J, Venstermans C, Kroon HM, Gielen J, Parizel PM, Bloem JL and De Schepper AM: Prevalence, extension and characteristics of fluid-fluid levels in bone and soft tissue tumors. Eur Radiol 16: 2644-2651, 2006.

6. Catalano P, Fang-Hui E and Som PM: Fluid-fluid levels in benign neurogenic tumors. AJNR Am J Neuroradiol 18: 385-387, 1997.

7. Al-Shraim M and Asa SL: The 2004 world health organization classification of pituitary tumors: What is new? Acta Neuropathol 111: 1-7, 2006.

8. Choi SH, Kwon BJ, Na DG, Kim JH, Han MH and Chang KH: Pituitary adenoma, craniopharyngioma and rathke cleft cyst involving both intrasellar and suprasellar regions: Differentiation using MRI. Clin Radiol 62: 453-462, 2007.

9. Warmuth-Metz M, Gnekow AK, Müller H and Solymosi L: Differential diagnosis of suprasellar tumors in children. Klin Padiatr 216: 323-330, 2004. 
10. Kurihara N, Takahashi S, Higano S, Ikeda H, Mugikura S, Singh LN Furuta S, Tamura H, Ishibashi T, Maruoka S and Yamada S: Hemorrhage in pituitary adenoma: Correlation of MR imaging with operative findings. Eur Radiol 8: 971-976, 1998.

11. Goel A, Shah A, Jhawar SS and Goel NK: Fluid-fluid level in pituitary tumors: Analysis of management of 106 cases. J Neurosurg 112: 1341-1346, 2010.

12. Nawar RN, AbdelMannan D, Selman WR and Arafah BM: Pituitary tumor apoplexy: A review. J Intensive Care Med 23: 75-90, 2008.

13. Liu ZH, Chang CN, Pai PC, Wei KC, Jung SM, Chen NY and Chuang CC: Clinical features and surgical outcome of clinical and subclinical pituitary apoplexy. J Clin Neurosci 17: 694-699, 2010.

14. Lenthall RK, Dean JR, Bartlett JR and Jeffree MA: Intrapituitary fluid levels following haemorrhage: MRI appearances in 13 cases. Neuroradiology 41: 167-170, 1999.

15. Bonneville F, Cattin F, Marsot-Dupuch K, Dormont D, Bonneville JF and Chiras J: T1 signal hyperintensity in the sellar region: Spectrum of findings. Radiographics 26: 93-113, 2006.

16. Semple PL, Webb MK, de Villiers JC and Laws ER Jr: Pituitary apoplexy. Neurosurgery 56: 65-73, 2005.

17. Kim DJ, Song YJ, Kim SJ, Park MK, Choi SS and Kim KU: Pituitary hemorrhage: Classification and related factors. J Korean Neurosurg Soc 46: 23-30, 2009.

18. Kinoshita Y, Tominaga A, Usui S, Arita K, Sugiyama K and Kurisu K: Impact of subclinical haemorrhage on the pituitary gland in patients with pituitary adenomas. Clin Endocrinol (Oxf) 80: 720-725, 2014.

19. Sarwar KN, Huda MS, Van de Velde V, Hopkins L, Luck S, Preston R, McGowan BM, Carroll PV and Powrie JK: The prevalence and natural history of pituitary hemorrhage in prolactinoma. J Clin Endocrinol Metab 98: 2362-2367, 2013.
20. Zhang F, Chen J, Lu Y and Ding X: Manifestation, management and outcome of subclinical pituitary adenoma apoplexy. J Clin Neurosci 16: 1273-1275, 2009.

21. Shim JH, Song YJ, Kim DC, Park MK, Choi SS and Kim KU: Silent adenomas of pituitary gland: It's immunohistochemical features and clinical characteristics. J Korean Neurosurgical Society 40: 330-335, 2006.

22. Onesti ST, Wisniewski T and Post KD: Clinical versus subclinical pituitary apoplexy: Presentation, surgical management and outcome in 21 patients. Neurosurgery 26: 980-986, 1990.

23. Shin JL, Asa SL, Woodhouse LJ, Smyth HS and Ezzat S: Cystic lesions of the pituitary: Clinicopathological features distinguishing craniopharyngioma, rathke's cleft cyst and arachnoid cyst. J Clin Endocrinol Metab 84: 3972-3982, 1999.

24. Binning MJ, Gottfried ON, Osborn AG and Couldwell WT: Rathke cleft cyst intracystic nodule: A characteristic magnetic resonance imaging finding. J Neurosurg 103: 837-840, 2005.

25. Byun WM, Kim OL and Kim D: MR imaging findings of rathke's cleft cysts: Significance of intracystic nodules. AJNR Am J Neuroradiol 21: 485-488, 2000.

26. Wang SS, Xiao DY, Yu YH, Jing JJ, Zhao L and Wang RM: Diagnostic significance of intracystic nodules on MRI in rathke's cleft cyst. Int J Endocrinol 2012: 958732, 2012.

27. Kim E: A rathke's cleft cyst presenting with apoplexy. J Korean Neurosurg Soc 52: 404-406, 2012.

28. Kunii N, Abe T, Kawamo M, Tanioka D, Izumiyama $H$ and Moritani T: Rathke's cleft cysts: Differentiation from other cystic lesions in the pituitary fossa by use of single-shot fast spin-echo diffusion-weighted MR imaging. Acta Neurochir (Wien) 149: 759-769, 2007. 